\title{
From politics to economics: new deal in cancer war
}

\section{J. Gligorov}

Service d'oncologie médicale, AP-HP, hôpital Tenon, université Paris-VI, F-75970 Paris, France Correspondance : gligorov@orange.fr

Si l'on se reporte à la définition d'un éditorial, il semble qu'il s'agisse de définir la ligne " politique " d'un journal ou d'une revue. Qu'il est donc difficile de définir la ligne éditoriale d'une revue dont c'est le dernier numéro ! En effet, à partir de 2009, ce supplément ne sera plus, faisant place à une actualité de recherche clinique intégrée régulièrement aux dossiers de la revue Oncologie et laissant le soin à l'INCa d'être le portail d'accès aux essais thérapeutiques...

Toutefois, mon esprit provocateur, l'évolution actuelle de la forme et du fond de notre petite sphère bleue me conduisent à penser que nous vivons une profonde mutation économique qui induira une mutation politique. Je m'explique : si l'on se réfère aux données politiques et économiques de la recherche médicale, il faut se rappeler que l'Hexagone a très clairement défini sa ligne politique de lutte contre le cancer en l'appuyant d'emblée sur une ligne budgétaire dédiée, notamment à la recherche clinique. Rappelons toutefois qu'en matière de politique économique du cancer, nos prédécesseurs d'outre-atlantique avaient déjà, sous le président Nixon, défini une stratégie comparable... avec des moyens différents. À l'aube du xxle siècle, on dépensait aux États-Unis cinq fois plus par habitant qu'en France pour la recherche [1]...

Voici pour la politique, mais qu'en est-il de l'économie ? Nos indices boursiers s'affolent, l'économie s'affole et malgré les euros et les dollars injectés, rien ne va plus. Le dopage n'est pas une solution à moyen terme, mais cela, les médecins sportifs le savaient depuis longtemps. Beaucoup plus angoissant pour un simple médecin de terrain comme moi est le retentissement de cet effondrement boursier sur la recherche en général et la recherche clinique en particulier. N'oublions pas l'apport considérable et incontournable des industries du médicament et du diagnostic dans la recherche clinique. Je ne juge pas des thématiques qu'ils choisissent - ce n'est ni mon rôle ni de ma compétence - mais indiscutablement, si la nouvelle donne économique mettait en péril cette ligne budgétaire, de nombreux axes de recherche, tant fondamentaux que cliniques, pourraient tout simplement être suspendus. Et pourtant, toute crise s'accompagne d'une révolution, et des changements importants nous amèneront à modifier nos pratiques de recherche dans les années à venir. Alors que nous sommes en train de récolter, en clinique, le fruit des investissements de la recherche fondamentale des années 1970 fondée sur l'exploration de l'ADN et la génomique, une nouvelle vague de découvertes fondamentales, portant sur les voies de signalisation, amène de nouveaux concepts et de nouvelles réflexions stratégiques sur la prise en charge du cancer. Peut-être devons-nous nous inspirer de la nature pour trouver une solution à ce nouveau défi économique du cancer [2] ? S'inspirant des voies de signalisation et des réseaux de communication au sein de la cellule, ce sont nos réseaux de soins et de recherche qui permettront de maintenir, malgré des restrictions budgétaires inévitables, une démarche de soins et de recherche clinique de qualité. Malraux disait que le $\mathrm{xxl}^{\mathrm{e}}$ siècle serait spirituel, dans le domaine de la recherche clinique en cancérologie, il ne pourra être qu'organisationnel ; c'est pourquoi, il semble logique que le travail initié par cette revue soit maintenant transmis à nos instances organisatrices. 
II ne me reste plus qu'à remercier l'ensemble des contributeurs de ces numéros spéciaux, les groupes coopérateurs, les collègues et amis médecins, chercheurs, rédacteurs d'articles et les membres du groupe Springer-Verlag qui ont soutenu ce projet.

Je vous souhaite à tous une recherche fructueuse et dirigée par un seul intérêt : celui de nos patients.

\section{Références}

1. https://www.e-cancer.fr/v1/fichiers/public/etude_economieducancer.pdf

2. Jones D (2008) Pathways to cancer therapy. Nat Rev Drug Discov 7: 875-6 